\title{
PREVENÇÃO DO USO DE DROGAS LÍCITAS E ILÍCITAS ENTRE ADOLESCENTES
}

\author{
Elisa Toffoli Rodrigues ${ }^{1}$ \\ Lara Miranda Kaminice ${ }^{2}$ \\ Mariana Barbosa Paranhos ${ }^{2}$ \\ Anne Kelly Azevedo Kil ${ }^{2}$ \\ Caroline Marchesoti Silvestre ${ }^{2}$ \\ Themissa Helena Voss ${ }^{2}$
}

\begin{abstract}
RESUMO: A percepção da redução contínua da idade de iniciação do uso de drogas sustenta a necessidade de abordar o tema dentro de uma perspectiva social e de saúde pública, nas quais as escolas são cenários privilegiados. O projeto "Prevenção do uso de drogas lícitas e ilícitas entre adolescentes" foi desenvolvido com alunos de uma escola de Ensino Médio da rede pública de ensino de Uberlândia, no ano de 2011. Foram realizadas oficinas com a finalidade de suscitar reflexões quanto aos impactos orgânico, financeiro e social do consumo de drogas lícitas e ilícitas. Os estudantes demonstraram interesse notável pelo tema, especialmente no tocante às drogas ilícitas, seus efeitos e consequências danosas comparativamente aos itens de consumo socialmente aceitos, como o álcool e o tabaco. Há ampla demanda para a manutenção e multiplicação de projetos semelhantes, a fim de reiterar o papel do consumo de psicoativos na violência social ou individual e fornecer ferramentas para que os adolescentes tomem decisões conscientes.
\end{abstract}

PALAVRAS-CHAVE: Drogas. Adolescentes. Prevenção. Extensão.

\section{Prevention of licit and illicit drugs use among adolescents}

\begin{abstract}
The perception of a steady reduction in the age of drug use initiation supports the need to address the issue within a social and public health perspectives, in which schools are privileged scenarios. The cycle of workshops was conducted with high school students from the public system of Uberlândia 2011 and relied on group dynamics in order to raise reflections on the organic, financial and social impacts of licit and illicit drugs consumption. Students have shown remarkable interest in the subject, especially in regards to illicit drugs, their effects and harmful consequences compared to the socially accepted consumable items such as alcohol and tobacco. There is a large demand for maintenance and multiplication of similar projects in order to reiterate the role of consumption of psychoactive drugs in individual or social violence and provide tools for adolescents to make educated decisions.
\end{abstract}

KEYWORDS: Drugs. Adolescents. Prevention. Extension.

\footnotetext{
${ }^{1}$ Mestranda em Saúde na Comunidade pela Faculdade de Medicina de Ribeirão Preto da Universidade de São Paulo, professora efetiva na Faculdade de Medicina da Universidade Federal de Uberlândia, coordenadora do Internato de Saúde Coletiva (elisa toffoli@yahoo.com.br).

2 Acadêmicas do curso de Medicina da Universidade Federal de Uberlândia, bolsistas no Programa Institucional de Bolsa de Extensão (PIBEX/2011)(lara_kaminice@hotmail.com; mariana-paranhos@hotmail.com; annekellyakil@yahoo.com.br; carolinemsilvestre@msn. com; themissa@hotmail.com).
} 


\section{INTRODUÇÃO}

O projeto "Prevenção do uso de drogas lícitas e ilícitas entre adolescentes", pautado nas diretrizes do Programa Institucional de Bolsas de Extensão (PIBEX-UFU/2011) e subsidiado pela Pró-reitoria de Extensão, Cultura e Assuntos Estudantis, da Universidade Federal de Uberlândia (PROEX-UFU), foi concebido a partir da percepção de que o uso de drogas por jovens está ocorrendo cada vez mais cedo, sendo a média de início de 12,5 anos, segundo Vieira e colaboradores (2007). Diante desse quadro de uso precoce, as ações de prevenção e conscientização fazem-se necessárias e de grande relevância para a redução do número de usuários no país.

Nesse sentido, Noto e Galduróz (1999) destacam três níveis de prevenção ao consumo de drogas. A prevenção primária consiste em ações que evitem o primeiro uso de uma determinada substância e a ocorrência de novos casos (OMS, 1992), dando-se, principalmente, por meio da disseminação de informações pelas comunidades. A prevenção secundária consiste em ações para evitar complicações em usuários esporádicos e que ainda não apresentam um nível alto de problemas (OMS, 1992). Já a prevenção terciária desenvolve-se no sentindo de reintegrar o usuário na sua comunidade, melhorando sua qualidade de vida (OMS, 1992), com abordagem sobre o tratamento do vício e auxílio durante as crises de abstinência, além de abordagem psicanalítica e medicamentosa.

Tendo em vista que a escola é um cenário privilegiado para realização de ações de prevenção e que a proposta de inclusão nos currículos de conteúdos relativos à prevenção do uso de drogas é, inclusive, uma das diretrizes da Política Nacional sobre Drogas (CONSELHO NACIONAL ANTIDROGAS, 2005), o projeto foi desenvolvido em uma escola da rede pública de ensino de Uberlândia, Minas Gerais, durante o ano de 2011, tendo como público- alvo estudantes do primeiro ano do Ensino Médio do turno noturno.

\section{As drogas como um problema de saúde pública}

O consumo de drogas na adolescência é corroborado por vários fatores como a suscetibilidade genética, a maneira como o jovem encaixa-se na vida social, a forma como ele interage com o meio e com seus familiares e o envolvimento emocional com seus problemas. Assim, se observa que os fenômenos relacionados ao uso de drogas nessa faixa etária são complexos e envolvem aspectos psíquicos e sociais.

Os fatores de risco para o uso de drogas incluem aspectos culturais, interpessoais, psicológicos e biológicos. São eles: a disponibilidade das substâncias, as leis, as normas sociais, as privações econômicas extremas; o uso de drogas ou atitudes positivas frente às drogas pela família, conflitos familiares graves; comportamento problemático (agressivo, alienado, rebelde), baixo aproveitamento escolar, alienação, atitude favorável em relação ao uso, início precoce do uso; susceptibilidade herdada ao uso e vulnerabilidade ao efeito de drogas (MARQUES; CRUZ, 2000, p. 35). 
Existe uma tendência mundial que aponta para o uso cada vez mais precoce de substâncias psicoativas, confirmando que a adolescência é uma época de exposição e vulnerabilidade ao consumo de drogas, quando ocorre frequentemente sua experimentação.

Para alguns adolescentes, o uso indevido de substâncias será apenas parte de seu processo de desenvolvimento, podendo cessar com seu amadurecimento. Outros, porém, desenvolverão um uso problemático, interrompendo o processo normal da adolescência, podendo trazer graves conseqüências para a vida desses indivíduos (SCIVOLETTO, 2001, p. 66).

Na pré-adolescência e adolescência, a idade do início do uso de drogas e seu padrão de consumo são fatores importantes na prevalência elevada do consumo dessas substâncias (VIEIRA et al., 2007).

Segundo Pechansky, Szobot e Scivoletto (2004), em um estudo que envolveu 107 grandes cidades brasileiras, cerca de $50 \%$ dos jovens entre 12 e 17 anos já consumiram drogas, sendo que, nesta porcentagem, 5,2\% é dependente de álcool. Assim, o trabalho se torna relevante por expandir o tema à faixa etária de grande vulnerabilidade para o consumo de drogas.

O uso na vida de álcool na população total foi de $68,7 \%$. Essa proporção se mantém mais ou menos estável para as diferentes faixas etárias, lembrando que, entre 12 e 17 anos, 48,3\% dos entrevistados já usaram bebidas alcoólicas (GALDURÓZ; CAETANO, 2004, p. 3).

As consequências, na vida das crianças e adolescentes, decorrentes do uso de drogas têm alto impacto social com prejuízo não somente aos jovens, mas à sociedade como um todo, que passa a ter honorários com tratamento e reabilitação dos dependentes, além do risco de contágio por outras doenças por meio, por exemplo, de drogas injetáveis como é o caso da AIDS (MARQUES; CRUZ, 2000).

Historicamente, a questão do uso abusivo e/ou dependência de álcool e outras drogas tem sido abordada por uma ótica predominantemente psiquiátrica ou médica. As implicações sociais, psicológicas, econômicas e políticas são evidentes, e devem ser consideradas na compreensão global do problema. Cabe ainda destacar que o tema vem sendo associado à criminalidade e práticas antissociais e à oferta de "tratamentos" inspirados em modelos de exclusão/separação dos usuários do convívio social (BRASIL, 2003, p. 7).

Em estudo realizado com jovens em situação de risco não usuários de drogas ilícitas, observouse que a disponibilidade de informações, com compreensão sobre as consequências do uso de drogas, além da estrutura familiar protetora eram fatores importantes para a negação à droga (SANCHEZ; OLIVEIRA; NAPPO, 2005). Portanto, as ações de prevenção ao uso de drogas são essenciais para conscientizar os jovens sobre os prejuízos sociais e as implicações negativas representadas pelo uso indevido e suas consequências. 


\section{METODOLOGIA}

A utilização de drogas é um problema de saúde pública e a exigência premente de ações de saúde voltadas à prevenção de seu uso e suas consequências para o organismo e a sociedade (BRASIL, 2003) compreenderam o foco central das ações extensionistas deste projeto. Assim, o projeto evidenciou a necessidade de se pautar na prevenção, tanto primária quanto secundária, estimulando os jovens a não serem usuários de drogas e aos que, por ventura, sejam drogaditos, a procurarem assistência na área da saúde.

O projeto foi desenvolvido em parceria com a Escola Estadual Messias Pedreiro, em Uberlândia, Minas Gerais, com estudantes que cursavam o primeiro ano do Ensino Médio no turno da noite, os quais, além de estarem entre o intervalo de idade de maior vulnerabilidade (PECHANSKY; SZOBOT; SCIVOLETTO, 2004), comportam-se como veiculadores de informações para terceiros, ampliando o espectro de ação do trabalho para a comunidade extra-acadêmica.

$\mathrm{Na}$ escola, aconteceram três encontros em cada sala, perfazendo um total de doze oficinas, que corroboram para a construção conjunta de conhecimento. O público atingido diretamente foi de 77 alunos. Considerando que cada participante das oficinas compartilhou as experiências e informações adquiridas a, no mínimo, um terceiro, acredita-se que o projeto tenha atingido um público indireto de, aproximadamente, 160 pessoas. Os assuntos abordados nas oficinas foram: "Álcool e suas consequências", "Drogas em geral: maconha, cocaína, crack, oxi, ecstasy e LSD" e, por fim, "Como procurar ajuda e a quem buscar".

Na primeira oficina, foi realizada uma dinâmica na qual os alunos escolhiam figuras, as descreviam e as relacionavam com o uso de drogas. Além disso, foi aplicado o questionário AUDIT - The alcohol use disorders identification test (BABOR et al., 2001). No segundo encontro, foi realizada uma dinâmica de colagem e uma discussão sobre a composição, os usos e os efeitos de diversas drogas. Na última oficina, ocorreu uma discussão acerca da rede de saúde mental na cidade de Uberlândia. No final de cada encontro, foi aplicado um questionário de avaliação das oficinas.

Por fim, como produto do projeto, foi elaborada e distribuída uma cartilha de orientação aos estudantes, contendo os serviços de saúde ou serviços da comunidade disponíveis no município para acolher usuários de álcool e outras drogas e abordando diversos tipos de drogas, seus efeitos e consequências, e as políticas públicas atuais sobre o assunto aos professores, para que os mesmos possam ampliar seus conhecimentos e sanar dúvidas dos estudantes.

\section{RESULTADOS E DISCUSSÃO}

A partir do questionário de sondagem do público alvo, observou-se que 77 alunos participaram das três oficinas realizadas, sendo que a maioria (54,5\%) pertencia à faixa etária de 14 a 16 anos, seguida pela faixa de 17 a 20 anos $(41,5 \%)$. 
No primeiro encontro com os estudantes, iniciou-se a discussão acerca do tema proposto, juntamente com uma dinâmica em que os participantes definiram o uso de drogas, sejam elas lícitas ou ilícitas, em uma palavra. Dentre os diversos adjetivos que surgiram, a maioria se tratava de características negativas (KESSLER et al., 2003) como: destruição, morte, doença, idiotice, inconsequência, loucura, violência; raras palavras de caráter positivo foram utilizadas, como: prazer e viagem. A partir daí, foi possível realizar uma discussão sobre os motivos que levariam as pessoas a experimentarem drogas, mesmo sabedoras das consequências negativas de seu uso e abuso (PECHANSKY; SZOBOT; SCIVOLETTO, 2004), instigando uma reflexão individual do custo benefício do envolvimento com elas.

Em seguida, realizou-se um bate-papo sobre o álcool, no qual foram abordadas informações sobre desde sua produção até a metabolização e os efeitos que o abuso desta droga pode causar no organismo e na sociedade. Notou-se que, por ser uma droga lícita e de uso muito prevalente na população, houve expressiva participação dos jovens, o que corrobora com a reflexão sobre a aceitabilidade do seu consumo na população brasileira (PECHANSKY; SZOBOT; SCIVOLETTO, 2004) mesmo entre adolescentes (BRASIL 2004).

Ao final do primeiro encontro, foi aplicado o questionário AUDIT (BABOR et al., 2001), um instrumento reconhecido e utilizado para rastreamento do consumo abusivo de álcool, apresentando sensibilidade e especificidade superiores aos demais testes de consumo autorreferido (MARTINS et al., 2008). O propósito desta ação foi estratificar o consumo de álcool entre os adolescentes, variando de consumo de alto risco (pontuação inferior a 8) até consumo abusivo com provável dependência química (acima de 20 pontos), que demandam diferentes estratégias de intervenção. Cada aluno realizou a soma de sua pontuação antes dos limites das faixas de consumo serem revelados, de modo a evitar alterações nas respostas, que pudessem mascarar os resultados do teste. Observou-se que a maioria dos estudantes não tinha consciência do seu próprio nível de consumo de álcool (11,7\% encontram-se na faixa de consumo de alto risco e provável dependência), uma vez que questionaram extensivamente as possíveis intervenções para cada grupo. Assim, o AUDIT consolida-se como uma ferramenta útil no rastreamento rápido e preciso do comprometimento do indivíduo com o álcool (MARTINS et al., 2008), revelando o risco do consumo, não só para o profissional que aplica o questionário, mas para o indivíduo que confronta valores palpáveis de sua ingestão de álcool, e a necessidade de alteração do hábito com intervenções educativas, como as realizadas nesta oficina, ou tratamento com especialista.

Na segunda oficina, por meio da dinâmica das figuras, foi possível discutir o tema a partir da realidade dos estudantes, perceber o consumo familiar de drogas, trabalhar seus anseios, focando na prevenção, e perceber a profundidade do conhecimento prévio dos alunos em relação ao assunto. Quanto ao consumo de drogas, tanto familiar, quanto individual, observou-se o álcool como o de maior utilização, seguido do tabaco. Entre os estudantes que participaram da oficina, o maior consumo de ambas as substâncias ocorreu entre as meninas, similar a outros estudos realizados no país (CARLINI et al., 2010). Os alunos envolveram-se na oficina de modo ativo, perguntando e expondo suas percepções.

Apesar da aparente confiança conquistada, em decorrência do intercâmbio gradual de informações entre alunos e estagiárias que conduziam as discussões, nenhum adolescente fez referência ao 
uso atual ou prévio de drogas ilícitas (por meio de questionário individual e não identificado). O resultado é questionado, já que, por ser ilegal, o consumo das drogas ilícitas pode não ter sido relatado por receio de seus usuários, em vista da retaliação social que os mesmos poderiam receber. Outro aspecto que reforça esta dúvida foi o maior interesse e melhor avaliação, por parte dos alunos, da oficina destinada à discussão sobre drogas ilícitas, especialmente a que tratou sobre a maconha.

Durante as oficinas que discutiram as drogas ilícitas, o objetivo foi conhecer aquilo que os alunos já possuíam de informações e aprofundar sobre as consequências e os malefícios de seu uso. Além disso, as discussões abrangeram a definição de cada droga, a sua ação, a comercialização e os riscos a curto e longo prazo. Procurou-se estimular a percepção dos alunos quanto à gravidade da situação de difusão das drogas na sociedade que interferem nos diferentes setores sociais. A apresentação sobre o oxi, em especial, elevou a curiosidade dos alunos, que ainda desconhecem tal droga e os males resultantes de sua utilização. Alguns se mantiveram em silêncio, por vezes, com expressão reflexiva e outros mostraram-se incomodados com o tema.

No questionamento sobre a produtividade da oficina, a grande maioria mostrou-se satisfeita e considerou o momento como de grande aprendizado, mostrando que faltam informações para embasar as escolhas dos jovens, tanto no início do uso de drogas, como na busca por tratamento.

O último encontro com cada turma teve o intuito de esclarecer aos adolescentes sobre os locais e os meios pelos quais eles poderiam buscar auxílio em caso de uso, abuso ou dependência de drogas. Pode-se perceber um conhecimento restrito da rede de saúde mental existente em Uberlândia, sendo citado como suporte para esse tipo de demanda: o Pronto Socorro do Hospital de Clínicas da Universidade Federal de Uberlândia (HC-UFU) e Unidades de Atendimento Integrado (UAIs). Alguns referiram conhecer as Unidades de Atendimento Primário à Saúde (UBSs ou UBSFs) e, pequena quantidade deles, sabia da existência do Centro de Atenção Psicossocial Álcool e Drogas (CAPS-AD).

Dessa forma, as oficinas serviram de alerta sobre a necessidade de realização de ações que viabilizem efetivamente a troca de conhecimento acerca do assunto, uma vez que, apesar da grande quantidade de informações veiculada pela mídia em geral, observa-se que os espaços de diálogo e de reflexões são pequenos. Além disso, pela relevância do tema discutido e pela busca de conhecimento, os alunos, em maioria, demonstraram, por meios dos questionários, aprovação para os encontros.

\section{CONSIDERAÇÕES FINAIS}

O trabalho corroborou com a percepção de que as drogas são um importante agravo em saúde pública e ratificou a relevância de ações de prevenção na comunidade escolar.

A participação dos jovens nas oficinas revelou grande interesse pelo tema, especialmente no tocante às drogas ilícitas, em contraste com o consumo autorreferido de álcool e tabaco, significativamente mais prevalente, provavelmente por sua aceitação social e cultural. Notouse, também, que os estudantes reconhecem e compreendem seu papel na sociedade e os efeitos 
negativos que as drogas podem causar ao organismo e à estrutura social.

O conhecimento sobre a rede de saúde mental no município de Uberlândia foi pequeno. A tendência de os jovens relatarem a busca de serviços de urgência e emergência, quando apresentam (ou se apresentassem) problemas relacionados a drogas, negligenciando a atenção primária, não difere dos demais agravos. Isso evidencia a necessidade de reforçar o papel de cada nível de densidade tecnológica, a fim de melhorar a funcionalidade e a eficácia do Sistema Único de Saúde (SUS).

Os resultados positivos na avaliação da aceitação e do aprendizado proporcionados pela intervenção reforçam-na como instrumento válido de promoção à saúde, visando à disseminação de informações sobre os transtornos orgânicos ou de adaptação social e o ônus financeiro proveniente do consumo de drogas lícitas e ilícitas - de maneira direta ou não -, suscitando reflexões sobre a inserção do indivíduo no contexto da sociedade e sua responsabilidade perante os demais.

Para a consolidação do enfrentamento às drogas na adolescência, compreende-se que diversos setores da sociedade deveriam se mobilizar e trabalhar em conjunto. Assim, compreende-se a limitação do projeto realizado, pois, além das ações de prevenção realizadas no ambiente escolar para o combate efetivo ao uso de drogas é imprescindível a melhoria da qualidade de vida desses jovens, com maior apoio familiar, maior acesso à saúde e outros equipamentos sociais, a exemplo de quadras de esportes, academias de musculação ou dança, aulas de teatro, música ou desenho, cursos de línguas ou computação e cursos técnicos profissionalizantes, dentre outros.

\section{REFERÊNCIAS}

BABOR, T. F. et al. AUDIT, the alcohol use disorders identification test: guidelines for use in primary care. Geneva: World Health Organization, 2001.

BRASIL. Ministério da Saúde. Secretaria Executiva. Coordenação Nacional de DST e AIDS. A política do Ministério da Saúde para a atenção integral a usuários de álcool e outras drogas. Série B. Textos Básicos de Saúde. Brasília, DF, 2003.

. Ministério da Saúde. Secretaria de Atenção à Saúde. Departamento de Ações Programáticas Estratégicas. Álcool e Redução de Danos: uma abordagem inovadora para países em transição. Série F. Comunicação e Educação em Saúde. Brasília, DF, 2004.

CARLINI, E. L. A. et al. VI Levantamento nacional sobre o consumo de drogas psicotrópica entre estudantes do ensino fundamental e médio das redes pública e privada de ensino nas 27 capitais brasileiras. SENAD, Brasília, 2010.

CONSELHO NACIONAL ANTIDROGAS. Resolução $n^{\circ}$ 3, de 27 de outubro de 2005, Aprova a Política Nacional sobre Drogas. Disponível em: < http://obid.senad.gov.br/portais/ OBID/conteudo/index.php?id_conteudo $=11178 \&$ rastro $=$ LEGISLA $\% C 3 \% 87 \% \mathrm{C3} \% 83 \mathrm{O} /$ Resolu\%C3\%A7\%C3\%B5es> Acesso em: 12 mar. 2011.

GALDURÓZ, J. C. F.; CAETANO, R. Epidemiologia do uso de álcool no Brasil. Rev. Bras. 
Psiquiatria, São Paulo, v. 26, Suppl. 1, p. 3-6, 2004.

KESSLER, F. et al. Pssicodinâmica do adolescente envolvido com drogas. Rev. Psiquiatria, Porto Alegre, v. 25, Suppl. 1, p. 33-41. 2003.

MARQUES, A. C. P. R; CRUZ, M. S. O adolescente e o uso de drogas. Rev. Bras. Psiquiatria, São Paulo, v. 22, Suppl. 2, p. 32-36, 2000.

MARTINS, R. A. et al. Utilização do Alcohol Use Disorders Identification Test (AUDIT) para identificação do consumo de álcool entre estudantes do ensino médio. R. Interam. Psicol., São Paulo, v. 42, n. 2, 2008.

NOTO, A. R.; GALDURÓZ, J. C. F. O uso de drogas psicotrópicas e a prevenção no Brasil. Ciência e Saúde Coletiva, Rio de Janeiro, v. 4, n. 1, p. 145-151, 1999.

OMS (Organização Mundial da Saúde). Reagindo aos problemas das drogas e do álcool na comunidade. São Paulo: Livraria Santos Editora, 1992.

PECHANSKY, F.; SZOBOT, C. M.; SCIVOLETTO, S. Uso de álcool entre adolescentes: conceitos, características epidemiológicas e fatores etiopatogênicos. Rev. Bras. Psiquiatria, São Paulo, v. 26, Suppl. 1, p.14-17, 2004.

SANCHEZ, Z. M.; OLIVEIRA, L. G; NAPPO, S. A. Razões para o não uso de drogas ilícitas entre jovens em situação de risco. Rev. Saúde Pública, São Paulo, v. 39, n. 4, p. 599-605, 2005.

SCIVOLETTO, S. Tratamento psiquiátrico de adolescentes usuários de drogas. In: FOCCHI, G. R. A et al. Dependência química: novos modelos de tratamento. São Paulo: Roca, 2001.

VIEIRA, D. L. et al. Álcool e adolescentes: estudo para implementar políticas municipais. Rev. Saúde Pública, São Paulo, v. 41, n. 3, p. 396-403, 2007.

Submetido em 3 de maio de 2012.

Aprovado em 8 de setembro de 2012. 